\title{
Avaliação da eficácia da azitromicina e pirimetamina em camundongos infectados por cepa cistogênica de Toxoplasma gondii
}

\author{
Evaluation of the efficacy of azithromycin and pyrimethamine, for treatment of experimental \\ infection of mice with Toxoplasma gondii cystogenic strain
}

\author{
Susana A.Z. Lescano ${ }^{1}$, Vicente Amato Neto ${ }^{2}$, Pedro P. Chieffi ${ }^{1,3}$, Rita C. Bezerra ${ }^{2}$, \\ Erika Gakiya ${ }^{2}$, Cláudio Santos Ferreira ${ }^{2}$ e Lúcia M.A. Braz ${ }^{2}$
}

\begin{abstract}
RESUM0
Foi avaliada a eficácia da administração prolongada de azitromicina e pirimetamina em camundongos infectados com cepa cistogênica de Toxoplasma gondii. Os animais foram inoculados intraperitonealmente com um cisto de T. gondii e, após 20 dias, divididos em quatro grupos: GI infectados não tratados, GII infectados e tratados concomitantemente com pirimetamina $(12,5 \mathrm{mg} / \mathrm{kg} / \mathrm{dia}$ ) e azitromicina (100m $\mathrm{m} / \mathrm{kg} / \mathrm{dia})$, GIII infectados e tratados com a mesma dose de pirimetamina e GIV infectados e tratados da mesma forma com azitromicina. 0 tratamento, via oral, estendeu-se por 120 dias; após este período os animais foram sacrificados e foi feita a contagem dos cistos no cérebro. Aassociação de ambos os medicamentos proporcionou melhores resultados, diminuindo a contagem de cistos no cérebro dos animais tratados de forma concomitante.
\end{abstract}

Palavras-chaves: Toxoplasma gondii. Cepa cistogênica. Infecção experimental. Azitromicina. Pirimetamina.

\begin{abstract}
The efficacy of prolonged administration of azithromycin and pyrimethamine was evaluated in mice experimentally infected with cystogenic strain of Toxoplasma gondii. The animals were intraperitoneally inoculated with one cyst of T. gondii and after 20 days were allocated into four groups: GI, infected without treatment; GII, infected and treated with the association of pyrimethamine $(12.5 \mathrm{mg} / \mathrm{kg} /$ day $)$ and azithromycin $(100 \mathrm{mg} / \mathrm{kg} /$ day $)$; GIII, infected and treated with the same dose of pyrimethamine; and GIV, infected and treated in the same way with azithromycin. The oral treatment lasted 120 days, after this period all the animals were sacrificed and the count of cysts in the brain was done. The association of both drugs provided the best results, by diminishing the cyst count in the brain of the animals treated in this way.
\end{abstract}

Key-words: Toxoplasma gondii. Cystogenic strain. Experimental infection. Azithromycin. Pyrimethamine.

Toxoplasma gondii, agente causal da toxoplasmose em hospedeiros imunocompetentes provoca infecção usualmente assintomática; porém em pacientes imunodeprimidos, submetidos a transplante de órgãos ou em tratamento por quimioterapia e, a partir da década de 80, infectados pelo vírus da imunodeficiência humana (HIV), a encefalite toxoplasmótica é a manifestação mais comum como resultante da reativação de uma infecção crônica passada e, se não tratada, pode ocasionar graves lesões cerebrais e mesmo ser fatal ${ }^{4691113}$.

0 tratamento padrão para a toxoplasmose adquirida é a combinação sinérgica de pirimetamina e sulfonamidas. № entanto, devido a sua toxicidade, a eficácia terapêutica desta combinação pode ser seriamente limitada nos imunodeprimidos, pois estes fármacos causam distúrbios colaterais expressivos.

1. Laboratório de Imunopatologia da Esquistossomose ( LIM 06) da Universidade de São Paulo, São Paulo, SP. 2. Laboratório de Investigação Médica do Departamento de Parasitologia ( LM 46) do Hospital das Clínicas da Faculdade de Medicina da Universidade de São Paulo, São Paulo, SP. 3. Faculdade de Ciências Médicas da Santa Casa de São Paulo, São Paulo, SP.

Suporte financeiro: Laboratórios Pfizer.

Endereço para correspondência: Dra. Susana Zevallos Lescano. Instituto de Medicina Tropical de São Paulo. R. Dr. Enéas de Carvalho Aguiar 470, Prédio II/2º andar, Cerqueira César, 05403-000 São Paulo, SP.

Tel: 11 3066-7063.

e-mail: chiong@uol.com.br

Recebido para publicação em 05/12/03

Aceito em 19/8/2004 
Novos agentes quimioterápicos menos tóxicos foram ensaiados em estudos clínicos, incluindo os antibióticos macrolídeos, entre eles a azitromicina que apresenta marcada afinidade pelos tecidos e meia-vida prolongada, ocasionando efeitos colaterais menos adversos ${ }^{18101516}$.

A pirimetamina, in vitro, inibe 0 crescimento dos taquizoítos em concentrações $\geq 0,05 \mathrm{mg} / \mathrm{L}$ e é parasiticida. Em modelos murinos de toxoplasmose aguda tem mostrado atividade protetora, porém a sua acumulação nos tecidos é retardada, pelo que deve ser administrada em combinação com sulfonamidas ou outras drogas. A azitromicina, cuja meia vida é prolongada e ocasiona menos efeitos adversos do que a eritromicina, em modelos de toxoplasmose aguda, só apresenta efeito moderado sobre a infecção no cérebro; esta droga também foi eficaz in vitro, porém não in vivo contra bradizoítos intracísticos ${ }^{5}$.

Estudos utilizando a associação azitromicina-pirimetamina em camundongos com toxoplasmose aguda mostraram a eliminação do parasita do sangue e pulmões resultando em proteção dos animais infectados e tratados ${ }^{38101113}$.

No presente trabalho, avaliamos a eficiência da azitromicina e pirimetamina, de forma isolada ou concomitante, na infecção murina experimental com cepa cistogênica de Toxoplasma gondii, produtora de infecções crônicas.

\section{MATERIAL E MÉTODOS}

Foram utilizados 60 camundongos BALB/C, machos, com 6 8 semanas, inoculados intraperitonealmente com um cisto de T. gondii ( cepa isolada na Universidade de Campinas, em 1978, e mantida por repiques sucessivos em camundongos) em 0,2ml de solução salina. Os animais foram divididos, após 20 dias de infecção, em quatro grupos de 15 animais cada:

GI: Infectados mantidos sem tratamento. GII: Infectados e tratados, de forma concomitante, com pirimetamina (DaraprimGlaxo Wellcome) na dose de $12,5 \mathrm{mg} / \mathrm{kg} /$ dia e azitromicina (Zitromax, Pfizer) na dose de 100mg/kg/dia. GIII: Infectados e tratados da mesma forma com pirimetamina. G IV: Infectados e tratados com a mesma dose de azitromicina.

0 tratamento, pela via oral, iniciou-se após 20 dias de infecção e extendeu-se por 120 dias, após os quais os animais sobreviventes foram sacrificados e foi feita a contagem de cistos na metade do cérebro de cada camundongo e a outra metade foi fixada para, posteriormente, ser submetida a cortes histológicos.

Para a contagem dos cistos, metade do cérebro de cada animal foi suspensa em PBS e passada dez vezes através de seringa de $5 \mathrm{ml}$ com agulha $22 \mathrm{~g}(0,9 \mathrm{~mm})$. 0 líquido resultante foi centrifugado a 3.000 rpm por 10 minutos à temperatura de $4^{\circ} \mathrm{C}$ e 0 número total de cistos em cada cérebro foi determinado pela leitura microscópica de sucessivas alíquotas de $25 \mu$ l, até esgotar 0 sedimento de cada tubo ${ }^{8}$.

Foram determinadas médias, desvio padrão e intervalos de confiança da contagem de cistos no cérebro dos animais (Média $\pm 2 x d p$ )

\section{RESULTAD0S}

A Tabela 1 abaixo mostra a taxa de sobrevivência dos camundongos nos quatro grupos estudados, bem como 0 número médio de cistos de T. gondii recuperados após necrópsia desses animais. A análise estatística desses resultados revelou que os animais tratados concomitantemente com azitromicina $\mathrm{e}$ pirimetamina apresentaram menor número de cistos no sistema nervoso central.

Tabela 1 - Percentagem de sobrevivência e número médio de cistos de Toxoplasma gondii recuperados dos camundongos dos quatro grupos estudados.

\begin{tabular}{|c|c|c|c|c|c|}
\hline \multirow[t]{2}{*}{ Grupo } & \multirow{2}{*}{$\begin{array}{c}\text { Animais no início } \\
\text { do experimento } \\
\mathrm{n}^{0}\end{array}$} & \multicolumn{2}{|c|}{$\begin{array}{c}\text { Animais } \\
\text { sobreviventes }\end{array}$} & \multirow[t]{2}{*}{$\begin{array}{l}\text { Número médio } \\
\text { de cistos }\end{array}$} & \multirow[t]{2}{*}{$\begin{array}{l}\text { Intervalos de Confiança } \\
\text { da contagem de cistos }\end{array}$} \\
\hline & & $\mathrm{n}^{0}$ & $\%$ & & \\
\hline GI & 15 & 11 & 73,3 & 174,4 & 151,0 a 197,0 \\
\hline GII & 15 & 12 & 80,0 & $14,8^{*}$ & 6,2 a 23,4 \\
\hline G III & 15 & 12 & 80,0 & 35,0 & 21,8 a 48,2 \\
\hline GIV & 15 & 14 & 93,3 & 78,6 & 56 a 101,2 \\
\hline
\end{tabular}

\section{DISCUSSÃ0}

Estudos terapêuticos utilizando a associação azitromicinapirimetamina em camundongos com toxoplasmose aguda mostraram a eliminação dos parasitas do sangue e pulmões de camundongos infectados, e redução da taxa de mortalidade, revelando proteção significativa dos animais tratados quando comparados com os controles infectados não tratados ${ }^{3}$. A azitromicina também é eficaz contra bradizoítos intracísticos in vitro mas a administração desta droga a longo prazo em camundongos cronicamente infectados não conseguiu reduzir o número médio de cistos no cérebro; no entanto foi observada redução na resposta inflamatória no cérebro dos animais infectados e tratados ${ }^{5}$.

Os resultados da presente comunicação indicam que tanto a azitromicina quanto a pirimetamina, mesmo administradas por um período de mais de três meses, não conseguiram produzir a cura parasitológica dos camundongos. Entretanto, observou-se decréscimo significativo no número de cistos entre os animais tratados com ambas as drogas quando comparado com os não tratados, indicando que o seu uso concomitante é mais eficaz do que a administração isolada. Bosch-Dresser e $\mathrm{Col}^{2}$, empregando essa associação em pacientes com lesões oculares de toxoplasmose, obtiveram resultados semelhantes aos da associação pirimetamina-sulfadiazina com relação à eficácia, porém observaram menor incidência de efeitos colaterais.

Emborajá existam alguns outros medicamentos com reconhecida atividade antitoxoplasma, como a clindamicina, 0 sulfametazol, 0 trimetoprim e a espirimicina, nenhum tem a eficácia da combinação antes citada e não apresentam atividade contra os cistos do parasita. É portanto prioritária na terapêutica da toxoplasmose, particularmente nos casos deinfectados pelo vírus $\mathrm{HIV}$, a necessidade dese encontrarem medicamentos que possam eliminar o microorganismo particularmente em sua forma cística ${ }^{1214}$. 


\section{REFERÊNCIAS BIBLIOGRÁFICAS}

1. Araujo FG, Guptill DR, Remington JS. Azithromycin, a macrolide antibiotic with potent activity against Toxoplasma gondii. Antimicrobial Agents and Chemotherapy 32:755-757, 1988.

2. Bosch-Driessen L, Verbraak F, Suttorp-Schulten M, Van Ruyven, Klok AM, Hoyng C, Rothova A. A prospective, randomized trial of pyrimethamine and azithromycin vs pyrimethamine and sulfadiazine for the treatment of ocular toxoplasmosis. American Journal of Ophthalmology 134: 34-40, 2002.

3. Braz LMA, Di Pietro A0, Amato-Neto V, França FOS. Avaliação da eficácia da azitromicina e da pirimetamina, usadas isolada ou associadamente, no tratamento de infecção experimental de camundongos pelo Toxoplasma gondii. Revista da Sociedade Brasileira de Medicina Tropical 32: 401-403, 1999.

4. Dannemann B, Mc Cutchan JA, Israelski D, Antoniskis D, Leport B, Luft B, Nussbaum J, Clumeck N, Morlat P, Chin J. Treatment of toxoplasmic encephalitis in patients with AIDS. A randomized trial comparing pyrimethamine plus clindamycin to pyrimethamine plus sulfadiazine. Annals of Internal Medicine 116: 33-43, 1992.

5. Derouin F. New pathogens and mode of action of azithromycin: Toxoplasma gondii. Pathology and Biology 43: 561-564, 1995.

6. Derouin F. Antitoxoplasmosis drugs. Current Opinion in Investigational Drugs 2: 1368-1374, 2001

7. Derouin F, Almadany R, Chau F, Rouveix B, Pocidalo JJ. Synergistic activity of azithromycin and pyrimethamine or sulfadiazine in acute experimental toxoplasmosis. Antimicrobial Agents and Chemotherapy 36: 997-1001, 1992

8. Dumas Jl, Chang R, Mermillod B, Piguet PF, Comte R. Evaluation of the efficacy of prolonged administration of azithromycin in a murine model of chronic toxoplasmosis. Journal of Antimicrobial Chemotherapy 34: 111 . 118, 1994.

9. Farthing C, Rendel M, Currie B, Seidlin M. Azithromycin for cerebral toxoplasmosis. The Lancet 339: 437-438, 1992.

10. Georgiev VS. Management of toxoplasmosis. Drugs 48: 179-188, 1994.

11. Godofsky EW. Treatment of presumed cerebral toxoplasmosis with azithromycin. The New England Journal de Medicine 330: 575-576, 1994.

12. Luft BJ, Remington JS. Toxoplasmic encephalitis in AIDS. Clinical Infectious Diseases 15: 211-222, 1992

13. Nahata MC, Koranyi KI, Gadgil SD, Hilligoss DM, Fouda HG, Gardner MJ. Pharmacokinetics of azithromycin in pediatric patients after oral administration of multiple doses of suspension. Antimicrobial Agents and Chemotherapy 37: 314-316, 1993.

14. Porter SB, Sande MA. Toxoplasmosis of the central nervous system in the acquired immunodeficiency syndrome. The New England Journal of Medicine 327: 1643-1648, 1992.

15. Renold C, Sugar A, Chave JP, Perrin L, Delavelle J, Pizzolato G, Burkhard P, Gabriel V, Hirschel B. Toxoplasma encephalitis in patients with the acquired immunodeficiency syndrome. Medicine 71: 224-239, 1992.

16. Wynn RF, Leen CL, Brettle RP. Azithromycin for cerebral toxoplasmosis in AIDS. The Lancet 341: 243-244, 1993. 